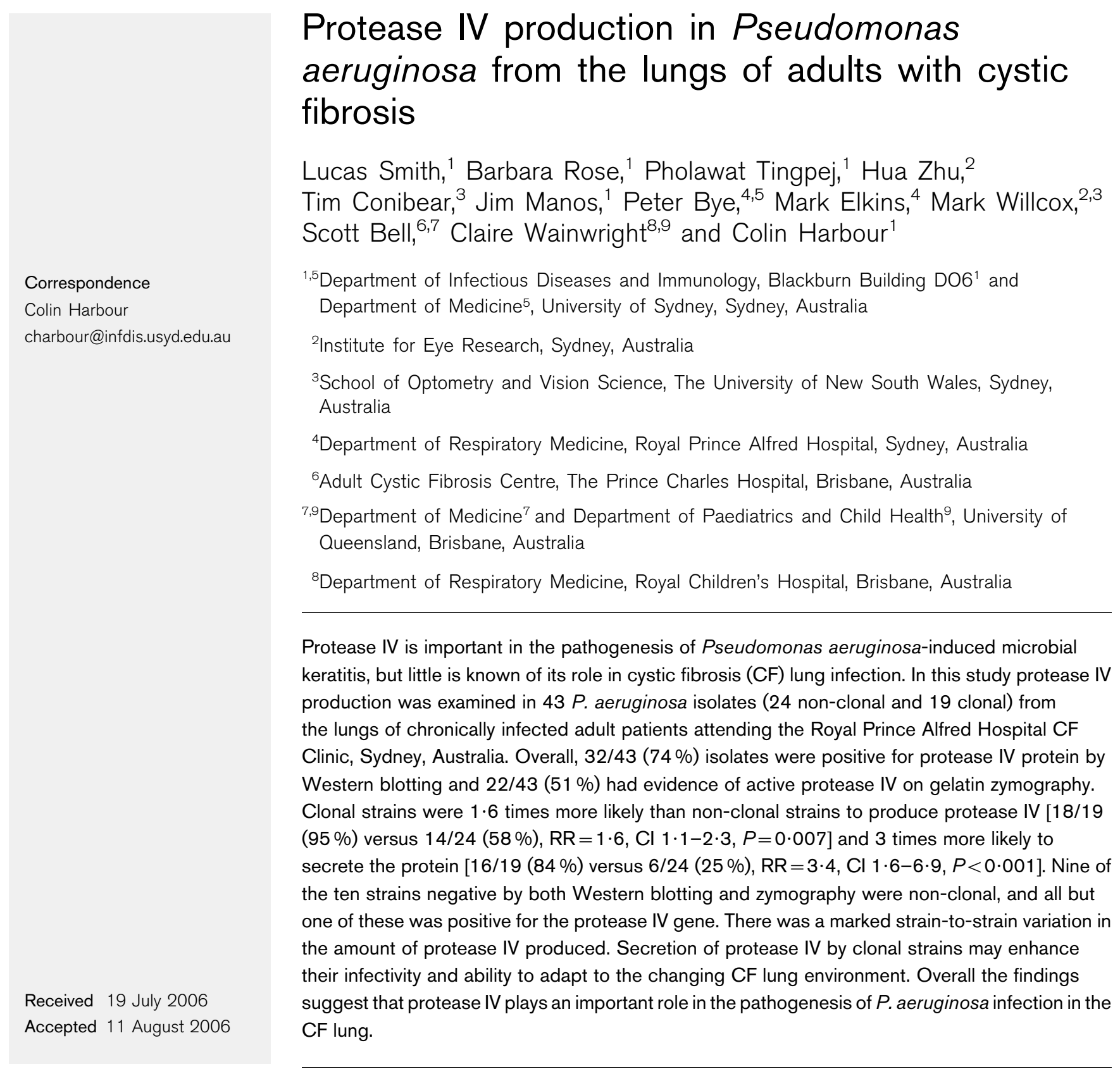

\title{
Protease IV production in Pseudomonas aeruginosa from the lungs of adults with cystic fibrosis
}

\author{
Lucas Smith, ${ }^{1}$ Barbara Rose, ${ }^{1}$ Pholawat Tingpej, ${ }^{1}$ Hua Zhu, ${ }^{2}$ \\ Tim Conibear, ${ }^{3}$ Jim Manos, ${ }^{1}$ Peter Bye, ${ }^{4,5}$ Mark Elkins, ${ }^{4}$ Mark Willcox, ${ }^{2,3}$ \\ Scott Bell, Claire Wainwright and Colin Harbour \\ ${ }^{5}$ Department of Infectious Diseases and Immunology, Blackburn Building D06 ${ }^{1}$ and \\ edicine ${ }^{5}$, University of Sydney, Sydney, Australia \\ Institute for Eye Research, Sydney, Australia \\ School of Optometry and Vision Science, The University of New South Wales, Sydney, \\ ${ }^{4}$ Department of Respiratory Medicine, Royal Prince Alfred Hospital, Sydney, Australia \\ es Hospital, Brisbane, Australia \\ Department of Medicine ${ }^{7}$ and Department of Paediatrics and Child Health ${ }^{9}$, University of \\ Department of Respiratory Medicine, Royal Children's Hospital, Brisbane, Australia
}

Protease IV is important in the pathogenesis of Pseudomonas aeruginosa-induced microbial keratitis, but little is known of its role in cystic fibrosis (CF) lung infection. In this study protease IV production was examined in $43 P$. aeruginosa isolates (24 non-clonal and 19 clonal) from the lungs of chronically infected adult patients attending the Royal Prince Alfred Hospital CF Clinic, Sydney, Australia. Overall, 32/43 (74\%) isolates were positive for protease IV protein by Western blotting and 22/43 (51\%) had evidence of active protease IV on gelatin zymography. Clonal strains were 1.6 times more likely than non-clonal strains to produce protease IV [18/19 (95\%) versus $14 / 24(58 \%), \mathrm{RR}=1 \cdot 6, \mathrm{Cl} 1 \cdot 1-2 \cdot 3, P=0 \cdot 007]$ and 3 times more likely to secrete the protein [16/19 (84\%) versus $6 / 24(25 \%), \mathrm{RR}=3 \cdot 4, \mathrm{Cl} 1 \cdot 6-6 \cdot 9, P<0 \cdot 001]$. Nine of the ten strains negative by both Western blotting and zymography were non-clonal, and all but one of these was positive for the protease IV gene. There was a marked strain-to-strain variation in the amount of protease IV produced. Secretion of protease IV by clonal strains may enhance their infectivity and ability to adapt to the changing CF lung environment. Overall the findings suggest that protease IV plays an important role in the pathogenesis of $P$. aeruginosa infection in the CF lung.

\section{INTRODUCTION}

Pseudomonas aeruginosa lung infection is a major cause of morbidity and mortality in patients with cystic fibrosis (CF) (Mellins et al., 1968). The pathogenesis of $P$. aeruginosa infection depends on a broad spectrum of virulence factors, including proteases. Proteases such as elastase (LasB), alkaline protease (AprA) and staphylolysin (LasA) have been characterized in many clinical settings, including the CF lung (Goodman \& Lory, 2004). Much less is known of

Abbreviations: $\mathrm{CF}$, cystic fibrosis; $\mathrm{Cl}$, confidence interval; $\mathrm{RR}$, relative risk. the distribution and properties of the recently described protease IV (PrpL) (O’Callaghan et al., 1996).

Protease IV, a $26 \mathrm{kDa}$ serine endoprotease (O'Callaghan et al., 1996; Engel et al., 1998a), is secreted by most $P$. aeruginosa isolates causing microbial keratitis and in association with other proteases has a major role in corneal virulence (O'Callaghan et al., 1996; Engel et al., 1997, 1998b; Wilderman et al., 2001). The virulence of protease IV in ocular infection has been attributed to the destruction of host proteins, including fibrinogen and components of the immune system (Engel et al., 1998a). Protease IV also degrades structural proteins such as elastin, facilitating bacterial adhesion and infection. Limited in vitro data 
suggest that protease IV may contribute to acute lung injury induced by $P$. aeruginosa through loss of surfactant function (Malloy et al., 2005). There is little information on protease IV secretion by clinical lung isolates.

P. aeruginosa has a unique relationship with the CF lung. Infections in early childhood are often acute and transient, and virulence factors, including the proteases, are expressed at high levels (Jain et al., 2004). However, over time the organism undergoes changes that enable it to persist indefinitely in the face of aggressive antibiotic therapy (Jain et al., 2004). These changes include down-regulation of virulence factors required for acute infection, such as the proteases, and an up-regulation of genes required for biofilm development.

It is widely accepted that most CF patients acquire unique (non-clonal) $P$. aeruginosa strains from the environment, although clonal $P$. aeruginosa strains have been reported with increasing frequency in recent years (Jones et al., 2001; McCallum et al., 2001; Scott \& Pitt, 2004). Two clonal strains, the Australian epidemic strain (AES)-1 (formerly known as the Melbourne epidemic strain, MES, m16 or PI) and AES-2 (PII) currently infect from 10 to $40 \%$ of patients in six CF clinics in eastern Australia (Armstrong et al., 2003; O'Carroll et al., 2004; H. Service, C. Lee, B. Rose, M. Elkins, P. Bye \& C. Harbour, unpublished data). The properties conferring the enhanced colonization of clonal strains have not been defined. The major aim of this study was to examine protease IV production in a series of clonal and non-clonal $P$. aeruginosa isolates from the chronically infected CF lung.

\section{METHODS}

Investigations were carried out on $43 P$. aeruginosa isolates from 43 patients attending the adult CF clinic at the Royal Prince Alfred Hospital, Sydney, Australia between 2001 and 2004. The study was approved by the Institutional Ethics Committee (approval X020320). Subjects gave informed consent to the work. The male to female ratio was $21: 22$; the mean age was 27 years and the age range $18-58$ years. All patients had been chronically infected with $P$. aeruginosa for a minimum of 4 years. Eighteen of the forty-three $(42 \%)$ isolates were derived from sputa collected at the time of exacerbation of symptoms and the remaining twenty-five (58\%) at routine clinic visits. The proportions within the clonal and nonclonal groups were similar. Genotyping by PFGE carried out using our published methods (Anthony et al., 2002) showed that 24 were non-clonal strains and that 19 were clonal (14 AES-1 and 5 AES-2). The proportion of clonal versus non-clonal strains was consistent with the distribution of strains in this clinic.

Bacteria were grown in tryptone soya broth to late exponential phase $\left(\mathrm{OD}_{600} \approx 1 \cdot 6\right)$ at $37^{\circ} \mathrm{C}$ with rapid agitation. The wound-derived $P$. aeruginosa reference strain PAO1 (Holloway et al., 1979) was used as a control. Supernatants were collected and filtered through a $0.22 \mu \mathrm{m}$ filter to remove any remaining cells. Cell suspensions were used for the detection of the $\operatorname{prpL}$ gene.

Western blotting. Western blotting to detect protein was carried out on $21 \mu \mathrm{l}$ supernatant using standard techniques. Blots were incubated overnight with rabbit polyclonal anti-protease IV antibody
(1:5000) (kindly donated by Brett Thibodeaux, Department of Microbiology, Immunology and Parasitology, Louisiana State University, Medical Center, New Orleans, LA, USA) and bands then imaged using a calibrated densitometer and QuantityOne software (Bio-Rad). All isolates were examined twice on different occasions.

Gelatin zymography. Gelatin zymography to assess protease IV activity was carried out on $21 \mu \mathrm{l}$ supernatant using $7 \cdot 5 \%$ polyacrylamide gels containing $0 \cdot 1 \%(\mathrm{w} / \mathrm{v})$ gelatin (Zhu et al., 2002). Zymograms were imaged using a calibrated densitometer and QuantityOne software (Bio-Rad). All isolates were tested twice on different occasions.

PCR for the protease IV gene. DNA was extracted from cell suspensions using the microLYSIS kit (Microzone). PCR was carried out using combinations of two sets of primers covering different parts of the protease IV gene: those described by Cabellero and colleagues (Caballero et al., 2004) (designated here as CabF and CabR) and the in-house designed primers $5^{\prime}$-TATTTCGCCGACTCCCTGTA-3' (TCF) and 5'-GAATAGACGCCGCTGAAATC-3' (TCR). The CabF/TCR combination yielded a $353 \mathrm{bp}$ product, the TCF/ CabR combination a $780 \mathrm{bp}$ product and the TCF/TCR combination a 752 bp product. PCRs were performed in $50 \mu \mathrm{l}$ mixes containing $25 \mu \mathrm{l}$ Green GoTaq master mix (Promega), 1.25 $\mu \mathrm{M}$ forward and reverse primers, $2.5 \mu \mathrm{l} 4 \%$ (v/v) glycerol and $2.5 \mu \mathrm{l}$ template. An annealing temperature of $60^{\circ} \mathrm{C}$ was used for each of the PCRs.

Dot hydridization. A DIG-labelled DNA probe was generated from the PAO1 strain using the CabF/TCR primer combination (above) according to the manufacturer's instructions (Roche Diagnostics). Bacterial DNA extractions were dot blotted onto Hybond-N+ membrane (General Electric Healthcare). Blots were then hybridized using DIG EasyHyb buffer (Roche Diagnostics). Following a standard high-stringency wash, hybrids were visualized by standard alkaline phosphatase colorimetric detection (Bio-Rad). Pseudomonas putida cells were used as a negative control.

Statistics. Tests of association between the production and secretion of protease IV and genotype were carried out using the Mantel-Haenszel method and Pearson's chi-square tests. $P$ values $<0.05$ were considered significant. Relative risk (RR) and $95 \%$ confidence intervals (CIs) were used to determine the strength of the associations.

\section{RESULTS AND DISCUSSION}

\section{Western blotting}

Overall 32 of the $43(74 \%)$ isolates and the PAO1 reference strain produced a band of $26 \mathrm{kDa}$ equivalent to that of the protease IV control (Fig. 1, Table 1). Although most positive samples produced a single band of the predicted size, a small proportion of samples with a strong band at $26 \mathrm{kDa}$ showed additional faint bands, including those at approximately 17 and $31 \mathrm{kDa}$. Other studies have also demonstrated these extraneous bands, with or without strong bands at $26 \mathrm{kDa}$, and have attributed them to the presence of precursor protease IV protein or to aggregation of the protein (Engel et al., 1998a; Traidej et al., 2003; Caballero et al., 2004). The $17 \mathrm{kDa}$ band has been reported to represent an enzymically active breakdown product of protease IV formed by autodigestion. Precursor protein may interfere with transport of the protease to the exterior of the cell. However, there was no association between the 
Zymography Control

Zymography

Western
blot

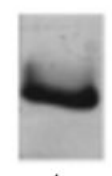

1
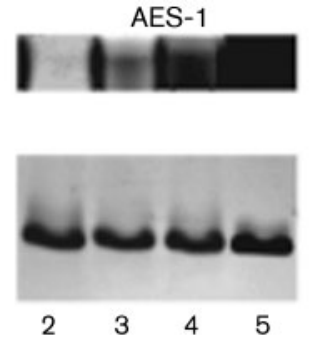
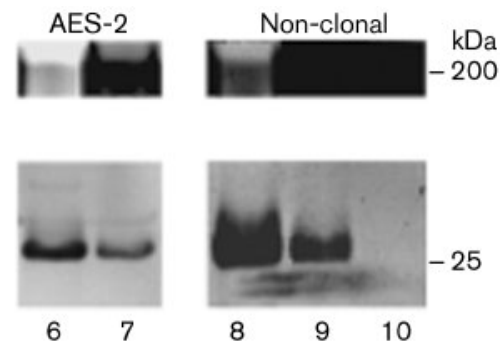

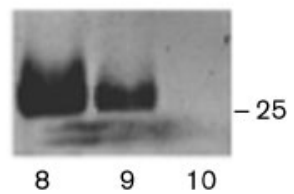

Fig. 1. Composite figure of Western blots and corresponding isolates on $1 \%$ gelatin zymograms indicating the presence of protease IV protein at $26 \mathrm{kDa}$ and evidence of protease IV activity at around $350 \mathrm{kDa}$. Lane 1, PAO1; lanes 2-5, AES-1; lanes 6-7, AES-2; lanes 8-10, non-clonal isolates. presence of extraneous bands on the Western blots and protease IV activity in the zymograms.

\section{Zymography}

Although the protease IV enzyme has a molecular mass of $26 \mathrm{kDa}$, it aggregates under mild SDS denaturing conditions, and is resolved at a molecular mass of $350 \mathrm{kDa}$ (Caballero et al., 2001). Overall, 22 of the 43 (51\%) isolates showed a diffuse region of activity at a molecular mass consistent with protease IV (Table 1). Ten of the eleven (91\%) zymography-negative isolates were also negative on the Western blot. As shown in Fig. 1, there was no relationship between the intensity of the band on the Western blot and protein activity on the zymogram.

\section{PCR for the prpL gene}

PCR was carried out on the ten isolates producing negative results by Western blotting and zymography. Nine of the ten were positive for at least one of the PCR assays confirming that the $\operatorname{prpL}$ gene is conserved among $\mathrm{CF}$ isolates; seven yielded a product using the $\mathrm{CabF} / \mathrm{TCR}$ primer combination and two using the TCF/CabR and TCF/TCR combinations. The remaining sample (non-clonal) was negative for all PCRs. All amplicons were of the size predicted from the primer design. The isolate with negative results on all PCRs was also negative by dot hybridization, suggesting that the prpL gene was lost or mutated.

\section{Protease IV expression: non-clonal versus clonal isolates}

The expression profile for protease IV in the non-clonal strains was markedly different from that in the clonal strains (Table 1). Collectively, clonal isolates were 1.6 times more likely to produce protease IV than non-clonal isolates $(P=0 \cdot 007)$, and were 3 times more likely to have active protein $(P<0 \cdot 001)$. Sixteen of eighteen $(89 \%)$ Western blot-positive clonal isolates had protease activity on the zymogram compared with only five of fourteen (36\%) Western blot-positive non-clonal isolates. All but one of the isolates testing negative by Western blotting and zymography fell into the non-clonal group. Recent studies have indicated that $P$. aeruginosa adapts to the hostile environment of the CF lung by progressively undergoing phenotypic or genotypic changes that down-regulate virulence factors required for acute infection and up-regulate factors required for biofilm formation (Smith et al., 2006). Our finding that only about half of the non-clonal isolates produced protease IV and less than one quarter secreted the protein suggests that protease IV is among the virulence factors usually down-regulated with chronic infection in the $\mathrm{CF}$ lung. Nonetheless, there was considerable strain-tostrain variation in the amount of protease IV protein produced by non-clonal strains, and a few produced higher levels of the protein than seen in any of the clonal isolates or PAO1. It has been reported that the expression of virulence factors is increased on exacerbation of symptoms (Grimwood et al., 1993). However, there was no relationship between protease IV production and the exacerbation of symptoms at the time of collection of the sputum.

Our finding that most isolates of both clonal strains secreted protease IV was of particular interest. The amount of protease IV produced by isolates of clonal strains was relatively uniform between and across the two groups, and in general was slightly less than that of PAO1. There is evidence in support of person-to-person spread of clonal strains, but acquisition from a common source has not been excluded. It is likely that the enhanced infectivity of clonal strains is multifactorial. However, we hypothesize that clonal strains, in contrast to non-clonal strains, have the ability to adapt for long-term survival in the CF lung by resisting modifications to the expression of virulence factors

Table 1. Production of protease IV by $P$. aeruginosa isolates from the CF lung

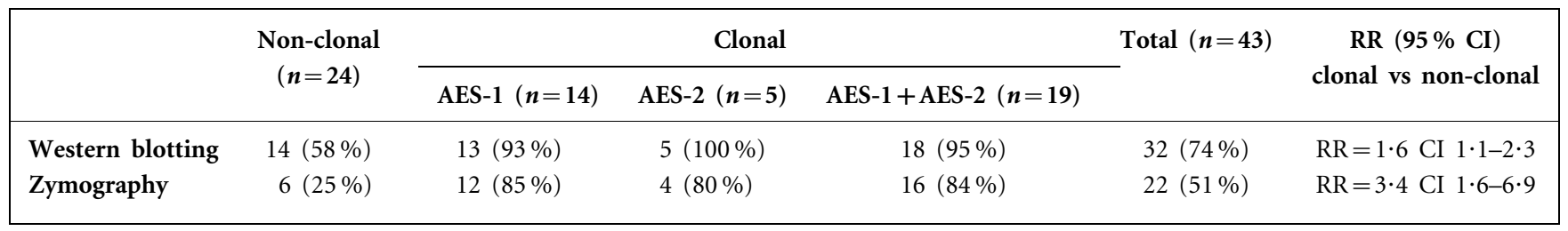


such as protease IV, and that this promotes infectivity and/ or facilitates person-to-person spread.

\section{CF lung versus corneal keratitis isolates}

Comparisons of data from non-clonal CF isolates with our findings from a study of microbial keratitis (Zhu et al., 2002) showed that the proportion of isolates with active protease IV was much higher among the keratitis group; $82 \%$ (14/17) of microbial keratitis isolates compared with $25 \%$ of nonclonal CF isolates. This discrepancy is likely to reflect both the different anatomical site and the nature of infection (acute in the case of keratitis versus chronic in the case of $\mathrm{CF})$.

In summary, this is the first study of the protease IV protein in a substantial number of $P$. aeruginosa isolates from the $\mathrm{CF}$ lung. This study has shown that the protease IV gene is highly conserved among CF lung isolates, which suggests that protease IV may have an important role in the pathogenesis of $P$. aeruginosa at this site. However, further studies will be needed to establish the contribution of protease IV to acute lung infection in young CF patients.

\section{ACKNOWLEDGEMENTS}

This work was carried out in the Department of Infectious Diseases, University of Sydney and the Institute for Eye Research and School of Optometry and Vision Science, The University of New South Wales, Sydney. We are grateful to the Australian Cystic Fibrosis Research Trust for their support.

\section{REFERENCES}

Anthony, M., Rose, B., Pegler, M. B. \& 8 other authors (2002). Genetic analysis of Pseudomonas aeruginosa isolates from the sputa of Australian adult cystic fibrosis patients. J Clin Microbiol 40, 27722778.

Armstrong, D., Bell, S., Robinson, M. \& 8 other authors (2003). Evidence for spread of a clonal strain of Pseudomonas aeruginosa among cystic fibrosis clinics. J Clin Microbiol 41, 2266-2267.

Caballero, A. R., Moreau, J. M., Engel, L. S., Marquart, M. E., Hill, J. M. \& O'Callaghan, R. J. (2001). Pseudomonas aeruginosa protease IV enzyme assays and comparison to other Pseudomonas proteases. Anal Biochem 290, 330-337.

Caballero, A., Thibodeaux, B., Marquart, M., Traidej, M. \& O'Callaghan, R. (2004). Pseudomonas keratitis: protease IV gene conservation, distribution, and production relative to virulence and other Pseudomonas proteases. Invest Ophthalmol Vis Sci 45, 522-530.

Engel, L. S., Hobden, J. A., Moreau, J. M., Callegan, M. C., Hill, J. M. \& O'Callaghan, R. J. (1997). Pseudomonas deficient in protease IV has significantly reduced corneal virulence. Invest Ophthalmol Vis Sci 38, 1535-1542.

Engel, L. S., Hill, J. M., Caballero, A. R., Green, L. C. \& O'Callaghan, R. J. (1998a). Protease IV, a unique extracellular protease and virulence factor from Pseudomonas aeruginosa. J Biol Chem 273, 16792-16797.
Engel, L. S., Hill, J. M., Moreau, J. M., Green, L. C., Hobden, J. A. \& O'Callaghan, R. J. (1998b). Pseudomonas aeruginosa protease IV produces corneal damage and contributes to bacterial virulence. Invest Ophthalmol Vis Sci 39, 662-665.

Goodman, A. L. \& Lory, S. (2004). Analysis of regulatory networks in Pseudomonas aeruginosa by genomewide transcriptional profiling. Curr Opin Microbiol 7, 39-44.

Grimwood, K., To, M., Semple, R. A., Rabin, H. R., Sokol, P. A. \& Woods, D. E. (1993). Elevated exoenzyme expression by Pseudomonas aeruginosa is correlated with exacerbations of lung disease in cystic fibrosis. Pediatr Pulmonol 15, 135-139.

Holloway, B. W., Krishnapillai, V. \& Morgan, A. F. (1979). Chromosomal genetics of Pseudomonas. Microbiol Rev 43, 73-102.

Jain, M., Ramirez, D., Seshadri, R. \& 7 other authors (2004). Type III secretion phenotypes of Pseudomonas aeruginosa strains change during infection of individuals with cystic fibrosis. J Clin Microbiol 42, 5229-5237.

Jones, A., Govan, J., Doherty, C., Dodd, M., Isalka, B., Stanbridge, T. \& Webb, A. (2001). Spread of a multiresistant strain of Pseudomonas aeruginosa in an adult cystic fibrosis clinic. Lancet 358, 557-558.

Malloy, J. L., Veldhuizen, R. A., Thibodeaux, B. A., O'Callaghan, R. J. \& Wright, J. R. (2005). Pseudomonas aeruginosa protease IV degrades surfactant proteins and inhibits surfactant host defense and biophysical functions. Am J Physiol Lung Cell Mol Physiol 288, L409-L418.

McCallum, S., Corkill, J., Gallagher, M., Ledson, M., Hart, C. \& Walshall, M. (2001). Superinfection with a transmissible strain of Pseudomonas aeruginosa in adults with cystic fibrosis chronically colonised by $P$. aeruginosa. Lancet 358, 558-560.

Mellins, R. B., Levine, O. R., Ingram, R. H. \& Fishman, A. P. (1968). Obstructive disease of the airways in cystic fibrosis. Pediatrics 41, 560-573.

O'Callaghan, R. J., Engel, L. S., Hobden, J. A., Callegan, M. C., Green, L. C. \& Hill, J. M. (1996). Pseudomonas keratitis. The role of an uncharacterized exoprotein, protease IV, in corneal virulence. Invest Ophthalmol Vis Sci 37, 534-543.

O'Carroll, M. R., Syrmis, M. W., Wainwright, C. E., Greer, R. M., Mitchell, P., Coulter, C., Sloots, T. P., Nissen, M. D. \& Bell, S. C. (2004). Clonal strains of Pseudomonas aeruginosa in paediatric and adult cystic fibrosis units. Eur Respir J 24, 101-106.

Scott, F. W. \& Pitt, T. L. (2004). Identification and characterization of transmissible Pseudomonas aeruginosa strains in cystic fibrosis patients in England and Wales. J Med Microbiol 53, 609-615.

Smith, E. E., Buckley, D. G., Wu, Z. \& 10 other authors (2006). Genetic adaptation by Pseudomonas aeruginosa to the airways of cystic fibrosis patients. Proc Natl Acad Sci U S A 103, 8487-8492.

Traidej, M., Caballero, A. R., Marquart, M. E., Thibodeaux, B. A. \& O'Callaghan, R. J. (2003). Molecular analysis of Pseudomonas aeruginosa protease IV expressed in Pseudomonas putida. Invest Ophthalmol Vis Sci 44, 190-196.

Wilderman, P. J., Vasil, A. I., Johnson, Z., Wilson, M. J., Cunliffe, H. E., Lamont, I. L. \& Vasil, M. L. (2001). Characterization of an endoprotease (PrpL) encoded by a PvdS-regulated gene in Pseudomonas aeruginosa. Infect Immun 69, 5385-5394.

Zhu, H., Thuruthyil, S. J. \& Willcox, M. D. (2002). Determination of quorum-sensing signal molecules and virulence factors of Pseudomonas aeruginosa isolates from contact lens-induced microbial keratitis. J Med Microbiol 51, 1063-1070. 